Résumés des conférences et travaux

\title{
Histoire des spectacles à l'époque contemporaine
}

Jean-Claude Yon

\section{OpenEdition \\ Journals}

Édition électronique

URL : https://journals.openedition.org/ashp/3912

DOI : 10.4000/ashp.3912

ISSN : 1969-6310

Éditeur

Publications de l'École Pratique des Hautes Études

\section{Édition imprimée}

Date de publication : 1 septembre 2020

Pagination : 288-289

ISSN : 0766-0677

\section{Référence électronique}

Jean-Claude Yon, "Histoire des spectacles à l'époque contemporaine », Annuaire de l'École pratique des hautes études (EPHE), Section des sciences historiques et philologiques [En ligne], 151 | 2020, mis en ligne le 09 juillet 2020, consulté le 06 juillet 2021. URL : http://journals.openedition.org/ashp/3912 ; DOI : https://doi.org/10.4000/ashp.3912 


\title{
HISTOIRE DES SPECTACLES À L'ÉPOQUE CONTEMPORAINE
}

\author{
Directeur d'études : M. Jean-Claude YoN
}

Programme de l'année 2018-2019: Histoire culturelle des spectacles au XIXe siècle.

Le groupe d'étudiants en master, de doctorants et d'auditeurs qui s'est constitué autour de Jean-Claude Yon a poursuivi, à l'occasion de cette cinquième année d'enseignement, l'exploration des spectacles en France au XIX ${ }^{\mathrm{e}}$ siècle menée depuis 2014 dans une perspective globale qui mêle les approches culturelle, sociale et politique. La méthode expérimentée les années précédentes a été reconduite : traiter un thème suffisamment large pour pouvoir aborder une grande variété d'aspects, adopter un rythme qui permet d'appréhender de la façon la plus concrète possible les réalités de la vie théâtrale, s'appuyer en permanence sur des documents dont la lecture et l'analyse détaillée constitue la matière même de l'enseignement.

Selon un usage qui permet d'intégrer les nouveaux arrivants, Jean-Claude Yon a commencé l'année par un thème particulier, en l'occurrence l'évocation de la carrière de Julia Bartet, « monstre sacré » de la Belle Époque qui a été une des principales inspirations de Proust pour La Berma. Puis a été inauguré un nouveau champ d'étude, à savoir l'histoire de l'opérette. Souvent dévalorisé, voire méprisé, ce genre à la fois dramatique et lyrique a été très peu étudié par le monde académique. Il est pourtant capital pour comprendre la société du second $\mathrm{XIX}^{\mathrm{e}}$ siècle dont il a marqué en profondeur la culture. Ce thème a été choisi par Jean-Claude Yon en relation avec la célébration en 2019 du bicentenaire de Jacques Offenbach - un bicentenaire dans lequel il s'est beaucoup impliqué, en France et à l'étranger. Cette histoire de l'opérette, au reste, sera menée sur plusieurs années tant le sujet reste largement à découvrir. JeanClaude Yon en a introduit l'étude par une réflexion sur le manque de débouchés des musiciens «dramatiques » au XIX ${ }^{\mathrm{e}}$ siècle, ce qui a conduit à consacrer des séances à l'Opéra national d'Adolphe Adam et au Théâtre-Lyrique.

Par ce biais, le parcours des deux créateurs de l'opérette, Florimond Ronger dit Hervé et Jacques Offenbach, prend tout son sens, par exemple l'activité du premier au théâtre Montmartre. La fondation du théâtre des Folies-Nouvelles et celle du théâtre des Bouffes-Parisiens ont été étudiées en détail et la lecture in extenso du prologue écrit en 1854 par Théodore de Banville pour la première de ces deux salles a été l'occasion d'aborder la poétique propre aux prologues d'ouverture, un genre précieux pour l'historien des spectacles. Pour tout ce qui concerne Offenbach, Jean-Claude Yon s'est appuyé sur les textes du compositeur qu'il a republiés au début de 2019 chez Actes Sud (M. Offenbach nous écrit. Lettres au Figaro et autres propos) avec le soutien du Palazzetto Bru Zane. La pièce d'ouverture de la salle d'hiver des Bouffes-Parisiens, $\mathrm{Ba}$-Ta-Clan, " chinoiserie musicale » d'une force politique incontestable, a été écoutée en entier et a fait l'objet d'un commentaire approfondi. Le choix de l'opérette a conduit en effet à multiplier cette année l'écoute d'extraits musicaux, ce qui a permis au passage des considérations sur l'évolution de l'interprétation de ce répertoire. 
Par ailleurs, du fait des séjours réguliers d'Offenbach dans la station thermale allemande de Bad Ems, a été étudiée la présence des spectacles dans ces lieux de villégiature - sujet que Chloé d'Arcy va traiter dans la thèse qu'elle a commencée à l'automne 2019 sous la direction de Jean-Claude Yon. Outre Hervé et Offenbach, le compositeur Léo Delibes, l'un des premiers à se lancer dans le nouveau genre de l'opérette, a fait l'objet de plusieurs séances. Autre compositeur d'opérette promis à un grand succès, Charles Lecocq a été également abordé. La fin du séminaire a en effet coïncidé avec la fin du Second Empire, ce qui a amené à traiter des effets de la guerre de 1870 sur la vie des spectacles et à revenir sur l'association entre le régime impérial et l'opérette, idée reçue encore courante de nos jours qui mérite d'être déconstruite et dont l'émergence a été étudiée.

Pour ne pas déroger à une habitude déjà prise, l'actualité théâtrale n'a pas été oubliée : Le Postillon de Lonjumeau d'Adam, repris en mars et avril 2019 à l'OpéraComique, a été étudié; de même - autre «tradition » du séminaire -, la dernière séance a eu lieu " hors les murs », en l'occurrence en arpentant le Paris théâtral du $\mathrm{XIX}^{\mathrm{e}}$ siècle, du Palais-Royal à la salle Ventadour, des Bouffes-Parisiens aux Variétés et au Gymnase. 